\title{
Citric Acid Production of Yeasts: An Overview
}

\author{
Bilge Sayın Börekçi ${ }^{*}$, Güzin Kaban², Mükerrem Kaya ${ }^{2}$
}

\section{Abstract \\ Background}

Citric acid, an intermediate product of the Krebs cycle, has a wide usage area in the food industry since it has some functions such as acidulant, flavouring agent, preservative and antioxidant. Although molds are the most commonly used microorganis$\mathrm{ms}$ in the citric acid production, it is known that there are significant advantages of using yeasts.

\section{Purpose and scope}

The microbial citric acid production mechanism needs to be well understood to make production more efficient. In this study, the yeasts used in the production, fermentation types and the factors affecting production were reviewed with studies.

\section{Methodology}

Although production of citric acid can be produced by chemical synthesis, the fermentation is preferred because of its low cost and ease of use. More than $90 \%$ of citric acid produced in the world is obtained by fermentation.

\section{Results}

Yarrowia lipolytica, Candida zeylanoides and Candida oleophila are evaluated for citric acid production with substrates such as molasses, glucose, sucrose and glycerol. On the other hand, there is great interest in developing processes with new substrates and/or microorganisms.

\section{Conclusion}

Although the microbial strain is an important factor, the factors such as carbon, phosphorus and nitrogen sources, aeration, the presence of trace elements and $\mathrm{pH}$ are also parameters affecting the production.

Keywords: citric acid, yeast, fermentation, Yarrowia, Candida

${ }^{1}$ Department of Food Engineering, Faculty of Engineering, Ardahan University, Ardahan 75000, Turkey

${ }^{2}$ Department of Food Engineering, Faculty of Agriculture, Atatürk University, Erzurum 25000, Turkey

Corresponding author: Bilge Sayın Börekçi E-mail: bilgesayin@ardahan.edu.tr ORCID: 0000-0002-1898-0428

DOI: 10.2478/ebtj-2021-0012

(C) 2021 Authors. This work was licensed under the Creative Commons Attribution-NonCommercial NoDerivs 4.0 License.

\section{Introduction}

Citric acid is an intermediate product that is colorless, odorless, readily soluble in water and alcohol, solid at room temperature and melts at $153^{\circ} \mathrm{C}$. It occurs when carbohydrates are oxidized to carbon dioxide in the Krebs cycle (1). Its molecular weight is $192.12 \mathrm{~g} / \mathrm{mol}$, which has three different values of $\mathrm{pKa}(3.13,4.76$ and 6.39) (2). Citric acid is the primary metabolic product formed in the Krebs cycle used by all aerobic organisms (3). The citric acid cycle is shown schematically in Figure 1.

Citric acid exists widely in nature and is present in fruits such as lemon, orange, pineapple, plum, peas, peach and also in bones, muscles and blood of animals (5). It was first isolated from lemon juice by the Swedish chemist Carl Scheele in 1784 (6). However, the first commercial citric acid was produced from calcium citrate in England in 1860 (7). In addition, it was reported that Wehmer was the first researcher who demonstrated that Penicillium species can produce citric acid in the medium containing sugar and inorganic salts in 1893 (8). In 1917, American food chemist James Currie discovered that certain 


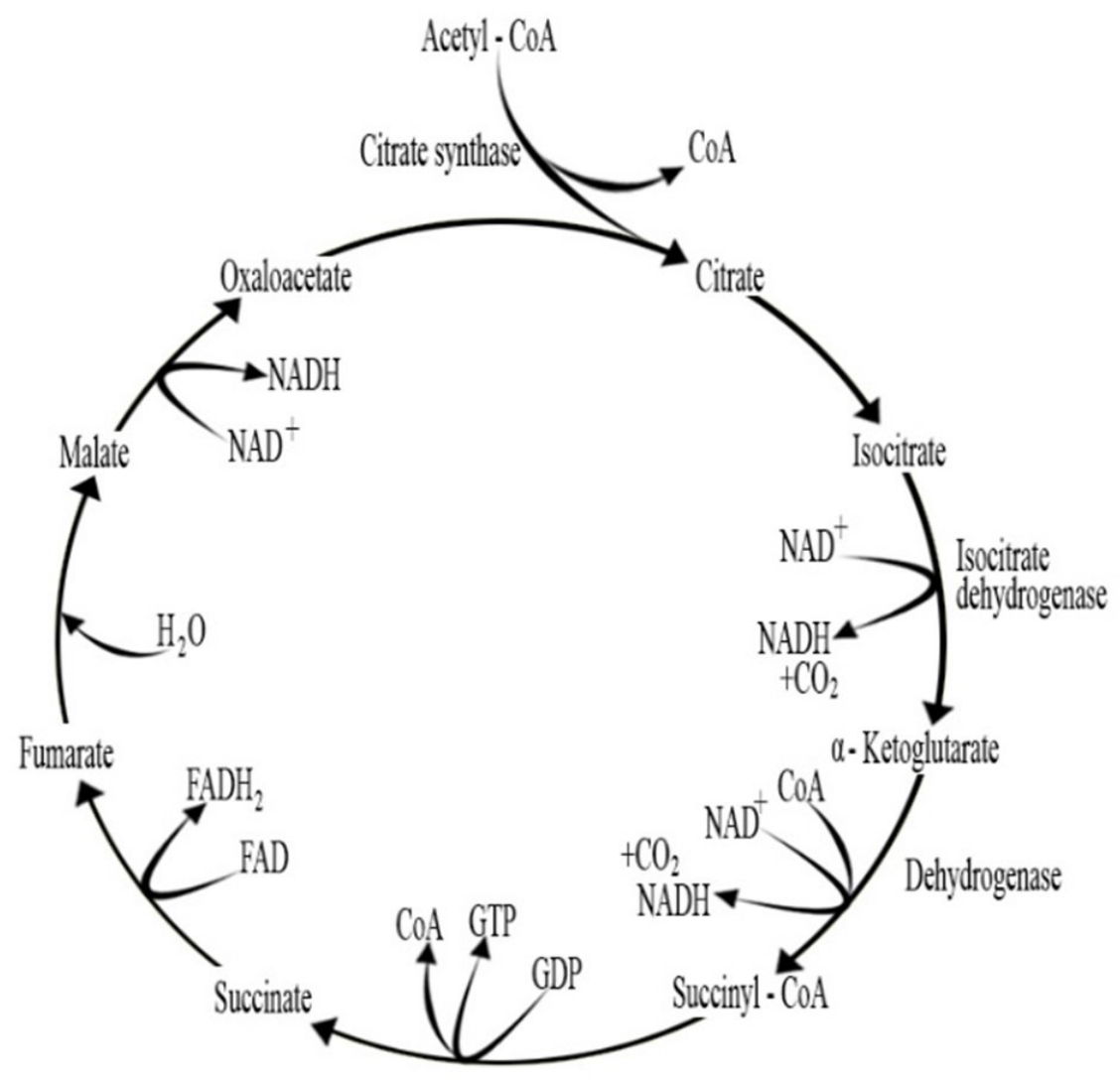

Figure 1. The citric acid cycle (4).

strains of Aspergillus niger can be used for the citric acid production, and Pfizer company started industrial production using this process (9).

Citric acid is accepted as GRAS by the JECFA (9) and is widely used in the food and pharmaceutical industries (10). $70 \%$ of the citric acid produced is used in the food industry, $12 \%$ in the pharmaceutical and the remaining $18 \%$ in other industries $(11,12)$. This organic acid has a wide area of application in the food industry due to its properties such as pleasant flavor, non-toxic agent, antioxidant (13), acidulant, flavouring agent (13-15), preservative and also emulsifier (14). It has also been used as a crosslinker in the production of detergent co-builder and biodegradable polymers in dishwasher cleaners over the past decade (16). The areas of usage of citric acid in the food industry and its functions are presented in Table 1. Citric acid production is considered a complex process that is affected by many metabolic and morphological changes (17). All the yeasts, molds and bacteria contain the citric acid cycle, but only some of them can increase citric acid production (18). It is reported that the annual production of citric acid is approximately 1.600 .000 metric tons and that the biggest producer in the world is China with a share of about $40 \%$ (12). It was reported that the global volume of citric acid is over two million tons and its production has increased by $5 \%$ annually (19). The global market for citric acid is estimated to reach 3.6 billion dollars by 2020. Moreover, an annual growth rate of $5.5 \%$ from 2015 to 2020 is also expected (20). Global demand continues to increase due to its applications in nanotechnology and tissue engineering (21).

\section{Citric Acid Production from Yeasts}

Citric acid is described as the most important organic acid produced in tonnage by microbial processes (27-29). In the world, more than $90 \%$ of citric acid production is obtained through fermentation $(6,10,23)$.

Candida, Hansenula, Pichia, Debaryomyces, Torulopsis, Kloekera, Trichosporon, Torula, Rhodotorula, Sporobolomyces, Endomyces, Nocardia, Nematospora, Saccharomyces, and Zygosaccharomyces are potential producers for citric acid (30). Nevertheless, some Candida species such as C. lipolytica, C. tropicalis, C. oleophila, C. intermedia, C. guilliermondii (30,31), C. paratropicalis, C. zeylanoides, C. catenulata, C. parapsilosis (14), C. citroformans (32), C. fibrae, C. subtropicalis (31), C. albicans, C. atmospherica, C. brumptii, C. chalmersii, C. hitachinica, C. krusei, C. melibiosa, C. mycoderma, C. pelliculosa, C. petrophilum, C. pulcherrima and C. rugosa (13) can be regarded to be among them.

At the end of the 1960s, it was determined that Yarrowia lipolytica showed quite high yields when n-alkanes were used as a substrate in citric acid production (33). However, it was also reported that glucose-containing media should be preferred rather than $\mathrm{n}$-alkanes due to the low production of isocitric acid (34). Using yeasts in the production has some advantages such as using many carbon sources due to the metabolic versatility and growth (2). Some studies about citric acid production by yeasts (strains, substrates, some fermentation conditions and 
Table 1. Areas of usage of citric acid in the food industry and its functions

\begin{tabular}{|c|c|c|}
\hline Industry & Functions & References \\
\hline Wines and ciders & $\begin{array}{l}\text { - Prevention of browning in some white wines } \\
\text { - Prevention of turbidity in wines and ciders } \\
\text { - } \mathrm{pH} \text { adjustment }\end{array}$ & $(10,22,23)$ \\
\hline Soft drinks and syrups & $\begin{array}{l}\text { - Provides tartness } \\
\text { - Enhancing the natural fruit flavor } \\
\text { - Acid regulation in carbonated and sucrose based beverages }\end{array}$ & \multirow[b]{2}{*}{$(10)$} \\
\hline Jellies, jams and preservatives & $\begin{array}{c}\text { - } \mathrm{pH} \text { adjustment } \\
\text { - Acid regulation } \\
\text { - Providing flavor, tang and tartness } \\
\text { - Increasing the efficiency of antimicrobial preservatives }\end{array}$ & \\
\hline Animal feed & - Feed complementation & $(10,23)$ \\
\hline Gelatin desserts & - $\mathrm{pH}$ adjustment & \multirow[b]{3}{*}{$(22)$} \\
\hline Animal fats and oils & - Producing synergetic effect with other antioxidants & \\
\hline Frozen fruits & $\begin{array}{c}\text { - Inactivation of oxidative enzymes } \\
\text { - Decreasing } \mathrm{pH} \\
\text { - Ascorbic acid protection }\end{array}$ & \\
\hline \multirow[b]{2}{*}{ Meat products } & - Antioxidant & $(24)$ \\
\hline & $\begin{array}{l}\text { - Prevention of coagulation or clotting of fresh blood in slaughterhou- } \\
\text { ses }\end{array}$ & \multirow{2}{*}{$(25)$} \\
\hline \multirow[b]{2}{*}{ Seafoods } & $\begin{array}{l}\text { - Prevention of discoloration and the development of off odors and } \\
\text { flavors by chelating trace metals }\end{array}$ & \\
\hline & $\begin{array}{l}\text { - Maintaining the stability and flavor by inactivating endogenous } \\
\text { enzymes } \\
\text { - Enhancing the action of antioxidants }\end{array}$ & $(26)$ \\
\hline Candies & $\begin{array}{l}\text { - Provides tartness } \\
\text { - Reducing sucrose inversion } \\
\text { - Producing dark color in hard candies } \\
\text { - Acid regulation }\end{array}$ & $(27)$ \\
\hline Dairy products & $\begin{array}{c}\text { - Emulsifier in ice creams and processed cheeses } \\
\text { - Acidifying agent in many cheese products } \\
\text { - Antioxidant }\end{array}$ & $(22,27)$ \\
\hline
\end{tabular}

the maximum amounts of citric acid) are presented in Table 2. Traditionally, Aspergillus niger has been used in the commercial production of citric acid from molasses, sucrose or glucose. Moreover, there is a great interest in various yeasts which have production capacity of citric acid from various carbon sources (35). The reasons for the preference of yeasts in the production are use of a variety of carbon sources, tolerance to certain conditions such as high concentrations of substrates, metal ions (36) and low oxygen levels (37) and fewer health hazards (38). Besides, the use of less refined substrates, the reduction in substrate and waste treatment, as well as the cost of product recovery (36) and easier genetic modifications by molecular techniques (39) are other important reasons for their preference. On the other hand, the biggest problem in the use of yeasts for production is considered to be the formation of isocitric acid $(36,37,40)$. This undesired by-product can not be easily obtained from plant tissue or by chemical synthesis (37). Isoc- itric acid was first found at growth of Y. lipolytica strains on n-alkanes in 1966 (13). After citric acid synthesis, isocitric acid is produced by the Krebs cycle, in a step catalyzed by aconitate hydratase (2). Isocitric acid is the structural isomer of citric acid. Excess synthesis of isocitric acid produced from cis-aconitate by the aconitase enzyme in the presence of iron ions occurs with high aconitase and citrate synthase activities and low isocitrate lyase and isocitrate dehydrogenase activities (41). The formation of this product varies depending on the strain used, substrate and current production conditions $(36,37)$. For this reason, it is emphasized that strains should be selected from those with the low isocitric acid capacity (42). It has been stated that wild-type strains can produce about $8-16 \%$ isocitric acid from carbohydrates or glycerol as the sole carbon source and $35-50 \%$ isocitric acid on the gluconeogenic substrates alkanes and renewable triglycerides, ethanol or acetate and also $35-67 \%$ isocitric acid on ethanol as the carbon source depending on 
cultivation conditions (37)

Citric acid is produced in the stationary phase of growth. The accumulation and release of citric acid in the cell are realized by different mechanisms (43). However, in a study carried out with $C$. oleophila, it was reported that there was a specific active transport mechanism for the earlier passage of citric acid through the cell membrane than isocitric acid (44).

\section{Fermentation Types Used in Citric Acid Production Submerged Fermentation (SmF)}

$\mathrm{SmF}$ is one of the methods used for commercial citric acid production (32). While SmF can be applied in batch, fed-batch or continuous systems, the batch system is the most preferred method $(6,27)$. Stirred tanks with $40-200 \mathrm{~m}^{3}$ capacity or larger airlift fermenters with $200-900 \mathrm{~m}^{3}$ capacity can be used for SmF (15). The bioreactors to be used for production should be acid-resistant because of the corrosive effect of citric acid and the decreasing $\mathrm{pH}$ value during fermentation (32). Tower fermenters are mainly preferred in terms of price, size and operation. Fermenters are also equipped with an aeration system to maintain the high dissolved oxygen level. Cooling can be performed by an external water film (27). This fermentation takes 6-8 days depending on growth of microorganism (31).

Anastassiadis and Rehm (44) researched citric acid production by C. oleophila ATCC 20177 under submerged continuous system using glucose. As a result, maximum citric acid concentration and citric/isocitric acid ratio were determined as 57.8 $\mathrm{g} / \mathrm{L}$ and 15.6 respectively at $\mathrm{pH}$ 5.0. In addition, Kamzolova et al. (45) investigated citric acid production capacity of Y. lipolytica $187 / 1$ with submerged batch fermentation. The strain was cultivated in a fermentor. They determined that $135 \mathrm{~g} / \mathrm{L}$ citric acid was obtained when rapeseed-oil was used (under nitrogen limitation conditions). In another study conducted on an acetate-negative mutant of Y. lipolytica Wratislavia K1, highest citric acid production $(110 \mathrm{~g} / \mathrm{L})$ was determined after $168 \mathrm{~h}$ of fed-batch cultivation with the total glycerol concentration of $250 \mathrm{~g} / \mathrm{L}$ (46).

SmF requires dilution of carbon source, pre-treatment of the appropriate nutrients and sterilization in line or in the bioreactor. This method offers advantages such as less space (32), sophisticated control mechanism (25), high productivity and yield, lower labour costs $(6,25,47)$ and lower contamination risk (6). On the other hand, the factors such as high-cost medium, sensitivity to metal inhibition, contamination risk and high amount of post-recovery wastewater generation are considered as disadvantages for this method (25).

\section{Solid State Fermentation (SSF)}

SSF fermentation is considered as an alternative method in which agro-industrial wastes can be evaluated for citric acid production $(27,47)$. This type of fermentation has an advantage about bioconversion of organic solid wastes through the production of biologically active metabolites both at the laboratory and industrial scale (48). Nevertheless, this fermentation type is a small-scale operation (15). Moreover, it is considered to be the simplest method for citric acid production $(6,10,27)$. This method was primarily applied through raw materials such as fruit wastes and rice bran $(6,10)$. The Erlenmeyer conical flasks, glass incubators, trays, rotating and horizontal drum bioreactors, packed-bed column bioreactor, single-layer packed-bed and multi-layer packed-bed systems are used for production with SSF (23). In this fermentation, the solid substrate is adjusted to $70 \%$ moisture level, the initial $\mathrm{pH}$ of the medium and the incubation temperature are adjusted to about 4.5-6.0 and $28-30^{\circ} \mathrm{C}$ respectively (depending on the microorganism used). Fermentation lasts for $96 \mathrm{~h}$ under optimum conditions (10). Although $A$. niger is the most commonly used microorganism in this method, it has been stated that some yeasts species such as Saccharomyces cerevisiae and Zygosaccharomyces bailii could also be used in this type of production (6). Imandi et al. (49) used the statistical experimental design for the optimization of citric acid production by Y. lipolytica NCIM 3589 in SSF using pineapple waste. Finally, the optimum conditions were found to be yeast extract $0.34(\mathrm{w} / \mathrm{w} \%)$, moisture content of the substrate $70.71(\%), \mathrm{KH}_{2} \mathrm{PO}_{4} 0.64(\mathrm{w} / \mathrm{w} \%)$ and $\mathrm{Na}_{2} \mathrm{HPO}_{4} 0.69$ $(\mathrm{w} / \mathrm{w} \%)$. Finally, they determined that citric acid concentration at these optimum conditions was $202.35 \mathrm{~g} / \mathrm{kg}$ ds.

The use of yeast with high nitrogen and phosphorus requirements is not appropriate due to the lower diffusion rate of nutrients and metabolites that occur at lower water activities in the SSF (10). This method involves difficulties in control of process parameters such as $\mathrm{pH}$, moisture, temperature, nutrients. Other disadvantage is higher recovery product costs due to the use of products with a higher impurity. On the other hand, this method has many advantages such as the use of simple technology, higher yield, wider range of low cost medium resembling natural habitat for various microorganisms, better oxygen circulation, less susceptibility to the inhibition of trace elements. Use of lower energy and cost requirements, lower risk of bacterial contamination and less post-recovery waste are other advantages of this method (25). It is also among the advantages that trace elements do not affect citric acid production as much as in $\mathrm{SmF}$ (10). As well as the advantages mentioned above, SSF requires less water usage in the upstream process and produces less waste water formation during the downstream process. On the other hand, it does not require the addition of some nutrients because of the use of agro-industrial residues (23).

\section{Factors Affecting the Production Temperature}

Although the optimum temperature for citric acid production varies depending on the organism and equipment used, it generally varies between 26 and $35^{\circ} \mathrm{C}(18)$. Previous studies about the production of citric acid have indicated that 28 and $30^{\circ} \mathrm{C}$ were usually selected as a fermentation temperature (Table 2). Moeller et al. (50) reported that the maximum citric acid concentration $(41 \mathrm{~g} / \mathrm{L})$ was obtained at $30^{\circ} \mathrm{C}$. They found that the citric acid concentration decreased rapidly at $34^{\circ} \mathrm{C}$ compared to at $32^{\circ} \mathrm{C}$. In another study, maximum citric acid was achieved at $28^{\circ} \mathrm{C}$ by glycerol-grown $Y$. lipolytica NG40/UV7 and citric 
acid concentration decreased gradually with temperature alteration (51). Anastassiadis and Rehm (52) studied the influence of temperature on citric acid production by $C$. oleophila and they determined that the maximum citric acid concentration $(63.5 \mathrm{~g} / \mathrm{L})$ and citric/isocitric acid ratio (28.8) were achieved at $30^{\circ} \mathrm{C}$.

\section{Carbon Sources}

The type and concentration of the carbon source are important for the process $(10,23)$ and yeasts can metabolize various carbon sources such as hydrocarbons, molasses, ethanol, vegetable oils, glycerol, galactose and glucose etc. (13). The presence of carbohydrates that are fermented more quickly by the microorganism is essential for production of citric acid $(10,23)$. The yields from glucose $(\mathrm{g} / \mathrm{g})$ decrease significantly at the concentration below $100 \mathrm{~g} / \mathrm{L}$, and that only small amounts of citric acid are produced at sugar concentration below $50 \mathrm{~g} / \mathrm{L}$ (53). Karasu-Yalçın et al. (54) reported that maximum citric acid concentration and productivity were achieved at $160 \mathrm{~g} / \mathrm{L}$ initial glycerol concentration, the highest product yield was obtained at $120 \mathrm{~g} / \mathrm{L}$ initial glycerol concentration for the Y. lipolytica 57 at the end of the $418 \mathrm{~h}$. Rane and Sims (55) detected that citric acid production by C. lipolytica Y 1095 depend on the initial glucose concentration and fermentation time. They obtained $13.6 \mathrm{~g} / \mathrm{L}$ citric acid with $50 \mathrm{~g} / \mathrm{L}$ initial glucose concentration at $27 \mathrm{~h}$. Additionally, citric acid concentration was increased to $78.5 \mathrm{~g} / \mathrm{L}$ with $150 \mathrm{~g} / \mathrm{L}$ initial glucose concentration at the end of the $128 \mathrm{~h}$. In a study conducted on the citric acid production by Y. lipolytica PR32, it was reported that when glucose concentration in the medium was $50 \mathrm{~g} / \mathrm{L}$, citric acid concentration formed was $24.76 \mathrm{~g} / \mathrm{L}$. However, when glucose concentration in the medium was $120 \mathrm{~g} / \mathrm{L}$, citric acid concentration was increased to $64.58 \mathrm{~g} / \mathrm{L}$ with a yield of $0.72 \mathrm{~g} / \mathrm{g}$ and a productivity of $0.45 \mathrm{~g} / \mathrm{L} / \mathrm{h}(56)$.

The fact that many natural substrates containing carbohydrate can be used more efficiently and afford in citric acid production is important (42). It has been indicated that the yeasts such as Y. lipolytica, C. guilliermondii and C. oleophila have the possibility to use a wider carbon source than fungi (especially, n-alkanes, glucose, raw glycerol, ethanol and galactose). Furthermore, yeasts can use less refined substrates (45). Hattori and Suzuki (57) used 10\% (w/v) n-alkane as substrate in a fermentor, and the citric acid concentration was determined as $87 \mathrm{mg} / \mathrm{ml}$ at pH 5.5 after $100 \mathrm{~h}$ for C. zeylanoides KY6166. It has been stated that molasses and invert sugar mixtures may also be used for citric acid production by yeast, although fructose assimilation levels of some yeasts are low (58). Glucose syrups from starch hydrolysis, sugar beet molasses and low-quality sugarcane by-products are the most preferred substrates in industrial productions. The yield of citric acid production increases depending on the initial sugar concentration in batch processes or glucose feeding rate in the chemostat process. It is reported that the highest productivities are usually obtained when sugar is used at the level of $14-22 \%$, since it was stated that this level leads to the suppression of ketoglutarate dehydrogenase (8). In a study, C. tropicalis ATCC 20115, C. oleophila ATCC 20373 ve ATCC 20177, C. zeylanoides ATCC 20367, Y. lipolytica ATCC 20237, ATCC 20346, ATCC 46330 and Saccharomycopsis lipolytica IFO1658 were examined for citric acid production. Fermentation was carried out in shake flasks. As a result, when sugarcane molasses was used as a substrate $91.4 \mathrm{~g} / \mathrm{L}$ of citric acid was achieved with C. zeylanoides ATCC 20367 (59).

As can be seen in Table 2, in previous studies, ethanol, canola oil, sunflower oil, rapeseed oil, glycerol, molasses, n-paraffin, fructose, sucrose, glucose and glucose hydrol were used as carbon sources for citric acid production by yeasts.

\section{Nitrogen and Phosphorus Sources}

Citric acid production is influenced by the concentration and nature of the nitrogen source $(10,23)$. Substances such as ammonium sulphate, ammonium chloride, malt extract, peptone, urea (47), yeast extract, beef extract, dry corn-steep, soybean (60), ammonium, sodium and potassium nitrate (22) are used as nitrogen source. However, Darvishi et al. (29) reported that urea was the best nitrogen source for citric acid production. On the other hand, some media such as molasses may not need additional nitrogen sources because they are rich in nitrogen (8). Ammonium nitrate at a concentration higher than $0.25 \%$ has been found to cause oxalic acid accumulation. But, it is stated that ammonium sulphate increases citric acid production without causing oxalic acid accumulation (22).

It was determined that citric acid production by yeasts starts after depletion of the nitrogen source (58). Nitrogen restriction coupled with a high concentration of glucose is very important for the citric acid production by yeasts. Citric acid is released by a specific energy-dependent transport system induced by intracellular nitrogen restriction (61). Because citrate synthase is negatively affected by the presence of ammonium ions (41). Souza et al. (62) determined that the citric acid production began after the stationary growth phase, when extracellular nitrogen was a limiting factor for yeast growth. Anastassiadis et al. (17) calculated citric acid concentration as $80 \mathrm{~g} / \mathrm{L}$ in fed-batch fermentation with Candida oleophila ATCC 20177. They remarked that ammonium nitrogen was the limiting factor for citrate formation. It was also stated that nitrogen content of $C$. oleophila biomass decreased during the production phase. According to obtained results, low intracellular nitrogen content and $\mathrm{NH} 4+$ concentration were found effective for the production.

Good adjustment of the high $\mathrm{C} / \mathrm{N}$ in production with yeasts plays an important role in citric acid biosynthesis. Nitrogen deficiency in the culture substrate leads to a rapid decrease in intracellular AMP. The excessive decrease of AMP concentration in the cell reduces the isocitrate dehydrogenase activity which converts isocitric acid to $\alpha$-ketoglutaric acid. Such conditions lead to intracellular accumulation of large quantities of isocitrate (63). Carsanba et al. (64) studied for the citric acid production by $Y$. lipolytica $\mathrm{K} 57$ in batch bioreactor. Finally, maximum citric acid yield (0.77 g/g glucose), titre $(72.3 \mathrm{~g} / \mathrm{L}$ 
Table 2. Maximum citric acid production by several yeast strains at different fermentation conditions

\begin{tabular}{|c|c|c|c|c|c|c|c|c|c|c|c|}
\hline Strain & Substrate & $\begin{array}{l}\text { ISC } \\
(\mathrm{g} / \mathrm{L})\end{array}$ & $\mathrm{N}$ and $\mathrm{P}$ sources & $\begin{array}{l}\text { Agi- } \\
\text { tation } \\
\text { rate } \\
\text { (rpm) }\end{array}$ & Cult. & Time & $\mathrm{pH}$ & $\mathrm{T}\left({ }^{\circ} \mathrm{C}\right)$ & $\begin{array}{l}\mathrm{CA}_{\max } \\
(\mathrm{g} / \mathrm{L})\end{array}$ & $\begin{array}{l}\text { ICA } \\
(\mathrm{g} / \mathrm{L})\end{array}$ & References \\
\hline${ }^{1} \mathrm{KY} 6166$ & & $\% 10$ & $\left(\mathrm{NH}_{4}\right)_{2} \mathrm{SO}_{4}, \mathrm{KH}_{2} \mathrm{PO}_{4}$ & 600 & & 100 & 5.5 & 30 & 87 & & (57) \\
\hline${ }^{2} \mathrm{NR}-$ & n-paraffin & 166 & $\begin{array}{c}\mathrm{KH}_{2} \mathrm{PO}_{4}, \\
\mathrm{Fe}\left(\mathrm{NO}_{3}\right)_{3} \cdot 9 \mathrm{H}_{2} \mathrm{O}\end{array}$ & 1000 & $\mathrm{~F}$ & 168 & 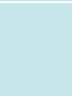 & 26 & 12 & & $(71)$ \\
\hline RL-Y-1095 & & 157 & $\begin{array}{l}\text { Urea, } \mathrm{KH}_{2} \mathrm{PO}_{4} \\
\mathrm{Fe}\left(\mathrm{NO}_{3}\right)_{3} \cdot 9 \mathrm{H}_{2} \mathrm{O}\end{array}$ & & & 120 & - & 20 & 9.8 & - & (18) \\
\hline${ }^{1}$ ATCC 20367 & Molasses & $\% 18$ & Molasses $+\mathrm{KH}_{2} \mathrm{PO}_{4}$ & 200 & S & 144 & 6.5 & & 91.4 & & (59) \\
\hline${ }^{3}$ ATCC 20177 & & 120 & & & & 139.5 & - & & 50.1 & 1.8 & $(17)$ \\
\hline A1Ce $201 / 1$ & & 240 & $\mathrm{NH}_{4} \mathrm{Cl}, \mathrm{KH}_{2} \mathrm{PO}_{4}$ & 1000 & & 58 & 5.0 & 30 & 74.2 & 3.9 & (79) \\
\hline${ }^{2} \mathrm{H} 222$ & & & & 1000 & & 92 & 6.0 & & 41 & 3.05 & $(50)$ \\
\hline${ }^{2} \mathrm{D} 1805$ & & 100 & $\mathrm{NH}_{4} \mathrm{NO}_{3}, \mathrm{KH}_{2} \mathrm{PO}_{4}$ & 950 & $\mathrm{~F}$ & 80 & 4.7 & & 95 & 10 & $(80)$ \\
\hline${ }^{2} \mathrm{Y} 1095$ & & & & 800 & & 128 & 5.5 & 27 & 78.5 & 7 & $(55)$ \\
\hline 2 & & 150 & $\mathrm{YE}, \mathrm{NH}_{4} \mathrm{Cl}, \mathrm{KH}_{2} \mathrm{PU}_{4}$ & 120 & S & 168 & 7.0 & 30 & 22.8 & & (1) \\
\hline${ }^{2}$ NCIM 3472 & & 100 & $\mathrm{NaNO}_{3}, \mathrm{KH}_{2} \mathrm{PO}_{4}$ & 160 & 3 & - & 5.5 & 30 & 8.4 & - & (75) \\
\hline${ }^{2} \mathrm{PR} 32$ & Glucose & 60 & $\begin{array}{c}\mathrm{YE}, \mathrm{CSL}, \mathrm{KH}_{2} \mathrm{PO}_{4}, \\
\mathrm{~K}_{2} \mathrm{HPO}_{4}\end{array}$ & 200 & & 240 & 6.5 & 28 & 111.1 & & $(56)$ \\
\hline${ }^{2}$ VKM Y-2373 & & 30 & $\begin{array}{c}\left(\mathrm{NH}_{4}\right)_{2} \mathrm{SO}_{4}, \mathrm{KH}_{2} \mathrm{PO}_{4} \\
\mathrm{~K}_{2} \mathrm{HPO}_{4}, \mathrm{Ca}\left(\mathrm{NO}_{3}\right)_{2}\end{array}$ & - & $\mathrm{F}$ & 144 & 6.0 & 30 & 85 & 4.7 & $(81)$ \\
\hline${ }^{2} \mathrm{~K} 57$ & & 110 & & 800 & & - & 6.0 & & 72.12 & & (64) \\
\hline $\begin{array}{c}{ }^{2} \text { ACA-DC } \\
50109\end{array}$ & & 149.5 & $\begin{array}{c}\mathrm{YE},\left(\mathrm{NH}_{4}\right)_{2} \mathrm{SO}_{4}, \\
\mathrm{KH}_{2} \mathrm{PO}_{4}, \mathrm{Na}_{2} \mathrm{HPO}_{4}\end{array}$ & - & S & 555 & 6.5 & 28 & 42.9 & - & (65) \\
\hline${ }^{2} \mathrm{M} 1$ & & 100 & & 200 & & 144 & & 29 & 27 & & $(82)$ \\
\hline${ }^{2} \mathrm{H} 222$ & & 150 & $\left(\mathrm{NH}_{4}\right)_{2} \mathrm{SO}_{4}, \mathrm{KH}_{2} \mathrm{PO}_{4}$ & & & 171 & 6.0 & \multirow{9}{*}{30} & 132.6 & 16.4 & (83) \\
\hline $\begin{array}{c}{ }^{2} \mathrm{H} 222-\mathrm{S} 4 \\
(\mathrm{p} 67 \mathrm{ICL} 1) \mathrm{T} 5\end{array}$ & Sucrose & 100 & $\begin{array}{l}\text { Proteose peptone, } \\
\mathrm{NH}_{4} \mathrm{Cl}, \mathrm{KH}_{2} \mathrm{PO}_{4}\end{array}$ & - & $\mathrm{F}$ & 191 & 6.8 & & 140 & 4 & (67) \\
\hline${ }^{2} \mathrm{~A}-101-1.14$ & $\begin{array}{l}\text { Glucose } \\
\text { hydrol }\end{array}$ & 400 & \multirow{7}{*}{$\mathrm{YE}, \mathrm{NH}_{4} \mathrm{Cl}, \mathrm{KH}_{2} \mathrm{PO}_{4}$} & 700 & $\mathrm{~F}$ & 80 & 5.5 & & 100 & - & (34) \\
\hline${ }^{2} 1.31$ & \multirow{7}{*}{ Glycerol } & 200 & & & & 132 & & & 124.5 & 3.9 & $(84)$ \\
\hline${ }^{2} 57$ & & 160 & & & S & 418 & 5.2 & & 32.8 & - & (54) \\
\hline $\begin{array}{c}{ }^{2} \text { Wratislavia } \\
\text { AWG7 }\end{array}$ & & 200 & & 600 & \multirow{5}{*}{$\mathrm{F}$} & 550 & \multirow{3}{*}{5.5} & & 97.8 & 5.1 & (85) \\
\hline $\begin{array}{c}{ }^{2} \text { Wratislavia } \\
1.31\end{array}$ & & \multirow{2}{*}{150} & & \multirow{4}{*}{800} & & & & & 92.8 & - & (72) \\
\hline $\begin{array}{c}{ }^{2} \text { Wratislavia } \\
\text { AWG7 }\end{array}$ & & & & & & - & & & 85.7 & 3.1 & $(86)$ \\
\hline${ }^{2} \mathrm{~A}-101-\mathrm{B} 56-5$ & & 100 & & & & 72 & 6.8 & & 57.15 & 0.4 & (87) \\
\hline${ }^{2} \mathrm{NG} 40 / \mathrm{UV7}$ & & 20 & $\begin{array}{l}\left(\mathrm{NH}_{4}\right)_{2} \mathrm{SO}_{4}, \mathrm{KH}_{2} \mathrm{PO}_{4} \\
\mathrm{~K}_{2} \mathrm{HPO}_{4}, \mathrm{Ca}\left(\mathrm{NO}_{3}\right)_{2}\end{array}$ & & & 192 & 5.0 & 28 & 115 & 4.6 & (51) \\
\hline
\end{tabular}




\begin{tabular}{|c|c|c|c|c|c|c|c|c|c|c|c|}
\hline Strain & Substrate & $\begin{array}{l}\text { ISC } \\
(\mathrm{g} / \mathrm{L})\end{array}$ & $\mathrm{N}$ and $\mathrm{P}$ sources & $\begin{array}{l}\text { Agi- } \\
\text { tation } \\
\text { rate } \\
\text { (rpm) }\end{array}$ & Cult. & Time & $\mathrm{pH}$ & $\mathrm{T}\left({ }^{\circ} \mathrm{C}\right)$ & $\begin{array}{l}\mathrm{CA}_{\max } \\
(\mathrm{g} / \mathrm{L})\end{array}$ & $\begin{array}{l}\text { ICA } \\
(\mathrm{g} / \mathrm{L})\end{array}$ & References \\
\hline $\begin{array}{l}{ }^{2} \mathrm{LGAM} \\
\mathrm{S}(7) 1\end{array}$ & \multirow{9}{*}{ Glycerol } & 120 & \multirow{5}{*}{$\begin{array}{c}\mathrm{YE},\left(\mathrm{NH}_{4}\right)_{2} \mathrm{SO}_{4}, \mathrm{KH}_{2} \mathrm{PO}_{4}, \\
\mathrm{Na}_{2} \mathrm{HPO}_{4}\end{array}$} & 185 & \multirow{3}{*}{$\mathrm{S}$} & 236 & $5-6$ & \multirow{5}{*}{28} & 35.1 & \multirow{2}{*}{-} & (88) \\
\hline $\begin{array}{l}{ }^{2} \text { ACA-DC } \\
50109\end{array}$ & & 164 & & 180 & & 600 & 6.1 & & 62.5 & & (89) \\
\hline $\begin{array}{l}{ }^{2} \text { NRRL YB- } \\
423\end{array}$ & & 40 & & 200 & & \multirow{2}{*}{240} & \multirow{3}{*}{6.0} & & 21.6 & 1.9 & $(90)$ \\
\hline $\begin{array}{l}{ }^{2} \mathrm{ACA}-\mathrm{DC} \\
50109\end{array}$ & & 104.9 & & $\begin{array}{l}200- \\
250\end{array}$ & $\mathrm{~F}$ & & & & 33.55 & \multirow{3}{*}{-} & $(91)$ \\
\hline $\begin{array}{l}{ }^{2} \mathrm{ACA}-\mathrm{YC} \\
5033\end{array}$ & & 120 & & 180 & \multirow[t]{2}{*}{ S } & 375 & & & 50.1 & & $(92)$ \\
\hline${ }^{2}$ NCIM 3589 & & 54.4 & $\mathrm{YE}, \mathrm{KH}_{2} \mathrm{PO}_{4}, \mathrm{Na}_{2} \mathrm{HPO}_{4}$ & 150 & & 72 & $5-6$ & \multirow{3}{*}{30} & 77.4 & & (93) \\
\hline $\begin{array}{l}{ }^{2} \text { Wratislavia } \\
\text { K1 }\end{array}$ & & 150 & \multirow{2}{*}{$\mathrm{YE}, \mathrm{NH}_{4} \mathrm{Cl}, \mathrm{KH}_{2} \mathrm{PO}_{4}$} & - & \multirow{10}{*}{$\mathrm{F}$} & 168 & \multirow[t]{2}{*}{5.5} & & 110 & 3.1 & $(46)$ \\
\hline${ }^{2} \mathrm{~A}-101-1.22$ & & 125 & & \multirow[b]{2}{*}{800} & & 1129 & & & 124.2 & 7.2 & $(94)$ \\
\hline${ }^{2} \mathrm{~N} 15$ & & 170 & $\begin{array}{c}\mathrm{YE},\left(\mathrm{NH}_{4}\right)_{2} \mathrm{SO}_{4}, \mathrm{KH}_{2} \mathrm{PO}_{4} \\
\mathrm{~K}_{2} \mathrm{HPO}_{4}, \mathrm{Ca}\left(\mathrm{NO}_{3}\right)_{2}\end{array}$ & & & 144 & \multirow{3}{*}{4.5} & \multirow{3}{*}{28} & 98 & 3.3 & (95) \\
\hline $\begin{array}{l}{ }^{2} \mathrm{VKM} \\
\mathrm{Y}-2373\end{array}$ & Ethanol & $<1.2$ & $\begin{array}{c}\left(\mathrm{NH}_{4}\right)_{2} \mathrm{SO}_{4}, \mathrm{KH}_{2} \mathrm{PO}_{4}, \\
\mathrm{~K}_{2} \mathrm{HPO}_{4}, \mathrm{Ca}\left(\mathrm{NO}_{3}\right)_{2}, \\
\text { yeast autolysate }\end{array}$ & \multirow[t]{2}{*}{ - } & & 145 & & & 116.8 & - & $(76)$ \\
\hline${ }^{2} \mathrm{~N} 1$ & & $<1$ & $\begin{array}{l}\left(\mathrm{NH}_{4}\right)_{2} \mathrm{SO}_{4}, \mathrm{KH}_{2} \mathrm{PO}_{4}, \\
\text { yeast autolysate }\end{array}$ & & & - & & & 21.6 & 6.4 & $(74)$ \\
\hline $\begin{array}{l}{ }^{2} \mathrm{VKM} \\
\mathrm{Y}-2373\end{array}$ & & $<10$ & $\begin{array}{c}\left(\mathrm{NH}_{4}\right)_{2} \mathrm{SO}_{4}, \mathrm{KH}_{2} \mathrm{PO}_{4} \\
\mathrm{~K}_{2} \mathrm{HPO}_{4} \\
\mathrm{Ca}\left(\mathrm{NO}_{3}\right)_{2} \cdot 4 \mathrm{H} 2 \mathrm{O}\end{array}$ & \multirow{3}{*}{800} & & 144 & \multirow[t]{2}{*}{5.0} & 29 & 22.6 & 90.5 & $(68)$ \\
\hline${ }^{2} \mathrm{NTG} 9$ & $\begin{array}{l}\text { Canola } \\
\text { oil }\end{array}$ & 15 & $\begin{array}{c}\mathrm{NH}_{4} \mathrm{CI}, \mathrm{KH}_{2} \mathrm{PO}_{4} \\
\mathrm{~K}_{2} \mathrm{HPO}_{4}\end{array}$ & & & 216 & & 30 & 137.5 & 49.2 & (78) \\
\hline${ }^{2} \mathrm{NG} 40 / \mathrm{UV7}$ & \multirow[t]{2}{*}{$\begin{array}{l}\text { Rapese- } \\
\text { ed oil }\end{array}$} & \multirow{3}{*}{20} & $\begin{array}{l}\mathrm{YE},\left(\mathrm{NH}_{4}\right)_{2} \mathrm{SO}_{4}, \mathrm{KH}_{2} \mathrm{PO}_{4}, \\
\mathrm{~K}_{2} \mathrm{HPO}_{4}, \mathrm{Ca}\left(\mathrm{NO}_{3}\right)_{2}\end{array}$ & & & 168 & 4.5 & \multirow{3}{*}{28} & 175 & 5.6 & $(35)$ \\
\hline${ }^{2} 187 / 1$ & & & \multirow{2}{*}{$\begin{array}{l}\left(\mathrm{NH}_{4}\right)_{2} \mathrm{SO}_{4}, \mathrm{KH}_{2} \mathrm{PO}_{4} \\
\mathrm{~K}_{2} \mathrm{HPO}_{4}, \mathrm{Ca}\left(\mathrm{NO}_{3}\right)_{2}\end{array}$} & \multirow{2}{*}{ - } & & \multirow{2}{*}{144} & 5.0 & & 135 & \multirow{3}{*}{ - } & $(45)$ \\
\hline${ }^{2} \mathrm{~N} 15$ & \multirow{3}{*}{$\begin{array}{c}\text { Sunf- } \\
\text { lower oil }\end{array}$} & & & & & & 4.5 & & 150 & & (96) \\
\hline $\begin{array}{l}{ }^{2} \mathrm{UOFS} \\
\mathrm{Y}-1701\end{array}$ & & 30 & $\mathrm{YE}, \mathrm{NH}_{4} \mathrm{Cl}, \mathrm{K}_{2} \mathrm{HPO}_{4}$ & 160 & \multirow[b]{2}{*}{ S } & 240 & 5.8 & 26 & 18.7 & & (97) \\
\hline${ }^{2}$ TEMYL3 & & 100 & $\begin{array}{c}\left(\mathrm{NH}_{4}\right)_{2} \mathrm{SO}_{4}, \mathrm{KH}_{2} \mathrm{PO}_{4} \\
\mathrm{~K}_{2} \mathrm{HPO}_{4}, \mathrm{Ca}(- \\
\left.\mathrm{NO}_{3}\right)_{2} \cdot 4 \mathrm{H}_{2} \mathrm{O}\end{array}$ & 150 & & 408 & 5.5 & 27 & 66.2 & 46.8 & (98) \\
\hline${ }^{2} \mathrm{SWJ}-1 \mathrm{~b}$ & $\begin{array}{l}\text { Waste } \\
\text { cooking } \\
\text { oil }\end{array}$ & 80 & \multirow{4}{*}{$\mathrm{YE}, \mathrm{NH}_{4} \mathrm{Cl}, \mathrm{K}_{2} \mathrm{HPO}_{4}$} & 250 & $\mathrm{~F}$ & 336 & - & 28 & 31.7 & 6.5 & (66) \\
\hline${ }^{2} \mathrm{~B} 9$ & Whey+a & $20 \mathrm{a}$ & & 150 & & 120 & 5.5 & 20 & 33.3 & 4.9 & (99) \\
\hline \multirow{2}{*}{${ }^{2} 57$} & Whey+ b & $150 \mathrm{~b}$ & & - & & 290 & 52 & 30 & 49.23 & 2.57 & $(100)$ \\
\hline & Fructose & 200 & & - & & 191 & 5.2 & 30 & 65.1 & 5.58 & (101) \\
\hline $\begin{array}{l}{ }^{2} \text { ACA-DC } \\
50109\end{array}$ & & $65 c$ & - & & S & - & $5-6$ & & 28.9 & & $(102)$ \\
\hline${ }^{2} \mathrm{ACA}-\mathrm{YC}$ & $+\mathrm{c}$ & $35 c$ & $\mathrm{YE},\left(\mathrm{NH}_{4}\right)_{2} \mathrm{SO}_{4}, \mathrm{KH}_{2} \mathrm{PO}_{4}$, & 180 & & 144 & 6.0 & 28 & 18.9 & - & $(103)$ \\
\hline 5033 & & $80 c$ & $\mathrm{Na}_{2} \mathrm{HPO}_{4}$ & & & 384 & $5-6$ & & 52 & & $(104)$ \\
\hline
\end{tabular}

${ }^{1}$ : Candida zeylanoides, ${ }^{2}$ : Yarrowia lipolytica, ${ }^{3}$ : Candida oleophila,

ISC: Initial substrate concentration, N: Nitrogen, P: Phosphorus, Cult: Cultivation, S: Shake flask, F: Fermenter, Time: Fermentation time, h: hours, T: Fermentation temperature, $\mathrm{CA}_{\max }$ : Maximum citric acid production, ICA: Isocitric acid, -: unspecified, $\bullet: \mathrm{ml} / \mathrm{L}$

YE: Yeast extract, CSL: Corn steep liquor, OMW: Olive mill wastewater

${ }^{\mathrm{a}}$ : Lactose, ${ }^{\mathrm{b}}$ : Fructose, ${ }^{\mathrm{c}}$ : Glucose 
citric acid) and productivity ( $0.04 \mathrm{~g} / \mathrm{g} \mathrm{h})$ were obtained with $\mathrm{C} / \mathrm{N}$ ratio of 367 . Papanikolaou et al. (65) examined the citric acid production of Y. lipolytica ACA-DC 50109 using different $\mathrm{C} / \mathrm{N}$ ratios $(110,138,172$ and $500 \mathrm{~mol} / \mathrm{mol})$. It was determined that when $\mathrm{C} / \mathrm{N}$ ratio and initial glucose concentration were increased, biomass and citric acid concentrations were increased also. Maximum citric acid concentration (42.9 g/L) and biomass $(7.2 \mathrm{~g} / \mathrm{L})$ were calculated with 149.5 initial glucose concentration and $500 \mathrm{C} / \mathrm{N}$ ratio. Additionally, Anastassiadis et al. (44) pointed out that biomass-specific nitrogen feed rate was the most effective factor influencing continuous citric acid production at yeast-based productions.

It was determined that the presence of phosphate in the medium has a significant effect on citric acid yield. Low phosphate levels have an enhancing effect on citric acid production and this effect is effective at the level of enzyme activity. Moreover, it has been stated that excess of phosphate leads to a decrease in carbon dioxide fixation and causes the formation of some sugar acids and the stimulation of growth (10). Additionally, production of citric and isocitric acid has usually been actualized under the restriction of yeast growth by nitrogen or other mineral components, such as sulphur, phosphorus and magnesium (45). Liu et al. (66) obtained citric acid from waste cooking oil using Y. lipolytica SWJ-1b and they indicated that the optimal concentration of ammonium sulphate added for the production was $0.2 \mathrm{~g} / \mathrm{L}$. In this condition, it was reported that 21.4 $\mathrm{g} / \mathrm{L}$ citric and $5.8 \mathrm{~g} / \mathrm{L}$ isocitric acid were obtained respectively. It was also found that extra ammonium sulphate was needed when waste cooking oil was used for citric acid production. Previous studies about the production of citric acid have shown that ammonium sulphate, ammonium chloride, yeast extract, potassium dihydrogen phosphate and dipotassium hydrogen phosphate were commonly used as nitrogen and phosphorus source (Table 2).

\section{pH}

The initial $\mathrm{pH}$ value should be adjusted and optimized according to the microorganism, substrate and production technique (23). There are many studies showing that the initial $\mathrm{pH}$ value of the medium for yeasts generally varies between 5.0 and 6.5 (Table 2). It is indicated that $\mathrm{pH}$ is an effective factor for the productions by $Y$. lipolytica and neutral $\mathrm{pH}$ values are favored for citric acid productions (41). Förster et al. (67) obtained citric acid by Y. lipolytica H222-S4 (p67ICL1) T5 strain using sucrose in the fermentation medium. Fermentation was carried out in a bioreactor. According to the data obtained, when the production medium was adjusted at values of $\mathrm{pH} 5.0,6.0$ and 6.8 , citric acid concentrations were calculated as 87,127 and $140 \mathrm{~g} / \mathrm{L}$ respectively. Moeller et al. (50) investigated the effect of $\mathrm{pH}$ value on the citric acid production on Y. lipolytica H222. As a result of the study, maximum citric acid $(24.91 \mathrm{~g} / \mathrm{L})$, selectivity of the production $(89.87 \%)$, yield ( $0.22 \mathrm{gCA} /$ gglucose $)$ and volumetric citric acid productivity $(0.27 \mathrm{gCA} / \mathrm{L} \mathrm{h})$ were calculated at pH 6.0. Kamzolova et al. (68) examined the role of different $\mathrm{pH}$ values on the production of citric acid by Y. lipolytica
VKM Y-2373 strain. According to obtained results, they stated that lowest $(12.1 \mathrm{~g} / \mathrm{L})$ and the highest $(40.15 \mathrm{~g} / \mathrm{L})$ citric acid concentration were obtained at $\mathrm{pH} 7.0$ and $\mathrm{pH} 4.5$, respectively.

\section{Aeration}

Oxygen is a very important parameter for microbial growth and metabolite production (69). It is reported that the formation of all organic acids increases under high aeration and that the high oxygen requirement results from the metabolic balance of citrate formation and the high sugar concentration used (70). The dissolved oxygen concentration also affects the concentration and type of organic compounds produced by yeasts (69). Since citric acid production is also an aerobic process, the oxygen plays an important role in production $(10,22,23)$. Citric acid yield increases depending on the increase in aeration rate, while the fermentation time is shortened (22). The interruption in aeration during batch fermentation was found to be quite harmful (10). It is very important to determine an optimal agitation rate for good production (100-180 g/L). On the other hand, high levels of agitation (1000 and $1200 \mathrm{rpm}$ ) can result in a shear stress on the cell-insoluble substrate interface on cell walls. Furthermore, it may lead to less cell-substrate interaction or decrease the cell viability (71). Another disadvantage of very high aeration rate $(>1.0 \mathrm{vvm})$ is the low partial pressure of dissolved $\mathrm{CO}_{2}$ in the broth. $\mathrm{CO}_{2}$ is important as a substrate for pyruvate carboxylase, but excessive aeration may cause some losses. Furthermore, it was emphasized that high $\mathrm{CO}_{2}$ levels were not found useful for the final concentrations of citrate and biomass (8). The aeration of the medium is applied at the same concentration throughout the fermentation process. It is found economically favorable to choose low oxygen levels at the beginning of fermentation. However, the combination of oxygen with air in the SmF increases citric acid production, but this is economically unviable. During the growth phase, high aeration levels may cause a large amount of foam formation. For this reason, it is emphasized that antifoaming agents should be added and mechanical defoamers should be used to solve this problem (23).

Rywińska et al. (72) used glycerol as substrate for citric acid production by Y. lipolytica Wratislavia AWG7 and Y. lipolytica Wratislavia 1.31. As an important result it was found that an increase in agitation and aeration rate had not any effect on dissolved oxygen concentration and citric acid production. Considering the research results, maximum citric acid concentration $(92.8 \mathrm{~g} / \mathrm{L})$ and yield $(0.63 \mathrm{~g} / \mathrm{g})$ were calculated by Y. lipolytica Wratislavia 1.31 at $0.24 \mathrm{vvm}$. Crolla and Kennedy (71) investigated the effect of fermentor agitation on citric acid production by $C$. lipolytica using n-paraffin. Eventually, it was determined that maximum citric acid concentration (12 g/L) was achieved using $1000 \mathrm{rpm}$ at 7 days with fed-batch fermentation. Sabra et al. (73) proved that control of dissolved oxygen concentration enhanced the citric acid production by Y. lipolytica in cultures grown on glucose in mono or dual substrate fermentations. Moreover, it was determined that dissolved oxygen concentration had no significant effect on the production 
which performed using glycerol.

As can be seen in Table 2, for citric acid production, agitation rates range from 120 to $200 \mathrm{rpm}$ for shake flask cultivation and 200 to $1000 \mathrm{rpm}$ for fermenter type.

\section{Alcohols}

It has been reported that ethyl alcohol, as well as carbohydrates, can be used in citric acid production and thus, high yields can be obtained (74). Lower alcohols such as methanol, ethanol, $\mathrm{n}$-propanol, isopropanol and methyl acetate inhibit citric acid production when they are used in pure form, but if added as crude carbohydrates increase the production. The alcohols have been reported to neutralize the negative effects of metals in citric acid production (generally in amounts about 1 to $5 \%)(10,23)$. The use of methanol and ethanol in the optimal amount varies depending on the strain and composition of the medium. It was stated that alcohols are effective on membrane permeability in microorganisms by affecting the phospholipid composition $(10,47)$. Pazouki et al. (75) reported that citric acid production by C. lipolytica NCIM 3472 was decreased with methanol addition to glucose based medium. On the other side, it was determined that using molasses $(35 \mathrm{~kg} / \mathrm{m} 3)$ with methanol $(3 \% \mathrm{v} / \mathrm{v})$ increased the citric acid concentration. Arzumanov et al. (76) studied citric acid production by mutant strain Y. lipolytica VKM Y-2373 with using ethanol as the sole carbon source. Maximum citric acid concentration was calculated as $116.8 \mathrm{~g} / \mathrm{L}$ in the batch system.

\section{Trace Elements}

Metal ions such as zinc, iron, copper, manganese and magnesium affect citric acid production (10). Some important integral components of metalloenzymes such as zinc and iron are involved in the yeast metabolism. Zinc is present as a component of dehydrogenases, aldolases, polymerases and proteases. It was determined that the need of zinc ions for yeasts increased in processes in which ethanol was used as a substrate. Moreover, it was indicated that NAD-dependent ADH activity under zinc restriction decreases significantly in different yeast species. Zinc is important because it acts as a stabilizer of the cell structure and some organelles (74).

Iron ions have been found to be effective on the activities of enzymes such as AIDH, $\mathrm{AH}$, catalase, peroxidases and the components of the mitochondrial electron transfer chain. On the other hand, it was stated that iron ions that are present at intracellular concentrations determine yeast requirements for oxygen (74). It has also been reported that iron restriction may cause the inactivation of aconitase in the tricarboxylic acid cycle (15). Additionally, the high efficiency of citric acid may be provided with a small excess of copper ions (10).

Finogenova et al. (74) examined the citric acid production by mutant Yarrowia lipolytica N1 using ethanol. It was proved that the production of citric and isocitric acid from ethanol required high concentrations of zinc and iron ions. At the same time, it was stated that intracellular iron concentration determined whether citric acid or isocitric acid was predominantly formed. In another study, it was proved that iron and copper salts had inhibition effects on citric acid production. It was reported that addition of manganese salt caused a decrease for the citric acid concentration. Maximum citric acid concentration $(41.63 \mathrm{~g} / \mathrm{L})$ was achieved by Y. lipolytica 57 by addition of $0.008 \mathrm{~g} / \mathrm{L}$ of zinc sulphate (77).

Manganese significantly affects the idiophase metabolism. While cell growth increases in the presence of manganese, sugar consumption and acidogenesis decrease drastically. Manganese deficiency results in the suppression of anaerobic and Krebs cycle enzymes, except for citrate synthase and this event leads to an overflow of citric acid. On the other hand, magnesium has been described as a metal involved both in citric acid production and cell growth (22).

Good et al. (78) studied the effect of micronutrients on citric acid production by $S$. lipolytica NTG9 using canola oil. As a result, it was determined that iron and zinc were found to have inhibitory effects on the production. Additionally, it was found that manganese stimulated citric acid synthesis. Liu et al. (66) investigated the effects of different magnesium amounts on the citric acid production obtained by Y. lipolytica SWJ1b using waste cooking oil. They reported that best concentration for the production was determined as $1 \mathrm{~g} / \mathrm{L}$ under non-optimized conditions.

\section{Conclusion}

The fact that the demand for citric acid with the developing industry is increasing from year to year makes citric acid production more important. The mechanism of microbial citric acid production needs to be well understood to meet the demand and to make production more affordable and efficient. The most species widely used in citric acid production is $A$. niger, however, yeasts play a prominent role in citric acid production. Yeasts can be preferred in the production because of some advantages such as high conversion ratios, tolerance to high substrate concentrations and less susceptibility to metal ion impurities and the tolerance to low oxygen levels. Y. lipolytica is a good producer in citric acid production, it is also thought that various yeast species, including C. zeylanoides and C. oleophila can be used in citric acid production.

\section{Abbreviations}

NAD: Nicotinamide adenine dinucleotide; FAD: Flavin adenine dinucleotide; GTP: Guanosine triphosphate; GDP: Guanosine diphosphate; $\mathrm{CO}_{2}$ : Carbon dioxide; GRAS: Generally Recognized as Safe; JECFA: Joint FAO/WHO Expert Committee on Food Additives; SmF: Submerged Fermentation; SSF: Solid State Fermentation; C/N: Carbon to Nitrogen ratio; AMP: Adenosine monophosphate; $\mathrm{AH}$ : Aconitate hydratase; $\mathrm{ADH}$ : Alcohol dehydrogenase; AIDH: Aldehyde dehydrogenase.

\section{Acknowledgements}

The authors received no financial support for this work.

\section{Conflict of interest}


The authors declare that they have no competing interests.

\section{References}

1. Abonama OM, Mahrous H, El AF, Hamza HA. Production of citric acid by Candida lipolytica under fermentation conditions using a Plackett-Burman design. Am J Food Nutr 2014;2(3):43-48. doi:10.12691/ajfn-2-3-2

2. Cavallo E, Charreau H, Cerrutti P, Foresti ML. Yarrowia lipolytica: A model yeast for citric acid production. FEMS Yeast Res 2017;17(8). doi:10.1093/femsyr/fox084

3. Höfer R. Sugar-and starch-based biorefineries. In: Pandey A, Höfer R, Taherzadeh M, Nampoothiri K, Larroche C, eds. Industrial Biorefineries\&White Biotechnology Elsevier; 2015:157-235.

4. Holness M, Sugden M, Naish J. Energy metabolism. In: Naish J, Court D, eds. Medical Sciences 2nd ed. Saunders Elsevier; 2015:57-100.

5. Ramesh T, Kalaiselvam M. An experimental study on citric acid production by Aspergillus niger using Gelidiella acerosa as a substrate. Indian J Microbiol 2011;51(3):289-293. doi:10.1007/s12088-011-0066-9

6. Swain M, Ray R, Patra J. Citric acid: Microbial production and applications in food and pharmaceutical industries. In: Vargas D, Medina J, eds. Citric Acid: Synthesis Properties and Applications Zagreb: Nova Science Publishers; 2012:97-118.

7. Abou-Zeid AZA, Ashy MA. Production of citric acid: A review. Agric Wastes 1984;9(1):51-76. doi:10.1016/01414607(84)90075-1

8. Max B, Salgado JM, Rodríguez N, Cortés S, Converti A, Domínguez JM. Biotechnological production of citric acid. Brazilian J Microbiol 2010;41(4):862-875. doi:10.1590/ S1517-83822010000400005

9. Shetty V. Production and optimization of citric acid by Aspergillus niger using molasses and corncob. Int J Pharm Pharm Sci 2015;(7):152-157.

10. Soccol CR, Vandenberghe LPS, Rodrigues C, Pandey A. New perspectives for citric acid production and application. Food Technol Biotechnol 2006;44(2):141-149.

11. Theron M, Lues J. Nature and composition of organic acids CRC Press; 2011.

12. Sato H., Kawaguti H. No Biotechnological production of organic acids. In: Bicas J., Maróstica M., Pastore G., eds. Biotechnological Production of Natural Ingredients For Food Industry 1st ed. Bentham Science Publishers; 2016:164-206.

13. Anastassiadis S, Morgunov I, Kamzolova S, Finogenova T. Citric acid production patent review. Recent Pat Biotechnol 2008;2(2):107-123. doi:10.2174/187220808784619757

14. Csutak O, Sarbu I. Genetically modified microorganisms: Harmful or helpful? In: Holban AM, Grumezescu AM, eds. Genetically Engineered Foods 6th ed. Elsevier; 2018:143-175.

15. Waites MJ, Morgan NL, Rockey JS, Higton G. Industrial microbiology: An introduction. In: Blackwell Science;
2001:144-164.

16. Rzechonek DA, Dobrowolski A, Rymowicz W, Mirończuk AM. Aseptic production of citric and isocitric acid from crude glycerol by genetically modified Yarrowia lipolytica. Bioresour Technol 2019;271:340-344. doi:https://doi. org/10.1016/j.biortech.2018.09.118

17. Anastassiadis S, Aivasidis A, Wandrey C. Citric acid production by Candida strains under intracellular nitrogen limitation. Appl Microbiol Biotechnol 2002;60(1-2):81-87. doi:10.1007/s00253-002-1098-1

18. Crolla A, Kennedy KJ. Optimization of citric acid production from Candida lipolytica Y-1095 using n-paraffin. J Biotechnol 2001;89(1):27-40. doi:10.1016/S01681656(01)00278-4

19. Morgunov IG, Kamzolova S V, Lunina JN. Citric acid production by Yarrowia lipolytica yeast on different renewable raw materials. Fermentation 2018;4(2). doi:10.3390/ fermentation 4020036

20. Markets and Markets. https://www.marketsandmarkets. com/PressReleases/citric-acid.asp. Published 2018. Accessed June 26, 2018.

21. Wang B, Chen J, Li H, Sun F, Li Y, Shi G. Pellet-dispersion strategy to simplify the seed cultivation of Aspergillus niger and optimize citric acid production. Bioprocess Biosyst Eng 2017;40(1):45-53. doi:10.1007/s00449-016-1673-y

22. Grewal HS, Kalra KL. Fungal production of citric acid. Biotechnol Adv 1995;13(2):209-234. doi:10.1016/07349750(95)00002-8

23. Soccol C, Vandenberghe LPS Rodrigues C, Pedroni Medeiros, AB Larroche C, Pandey A. Production of organic acids. In: Pandey A, Soccol C, Larroche C, eds. Current Developments in Solid-State Fermentation New Delhi: Asiatech Publishers; 2008:205-230.

24. Madhavi DL, Singhal RS, Kulkarni PR. Technological aspects of food antioxidants. In: Madhavi D., Deshpande S., Salunkhe D., eds. Food Antioxidants: Technological: Toxicological and Health Perspectives New York: Marcel Dekker; 1995:159-226.

25. Dhillon GS, Brar SK, Verma M, Tyagi RD. Recent advances in citric acid bio-production and recovery. Food Bioprocess Technol 2011;4(4):505-529. doi:10.1007/s11947-0100399-0

26. Sahasrabudhe NA, Sankpal N V. Production of organic acids and metabolites of fungi for food industry. In: Khachatourians GG, Arora D., eds. Applied Mycology and Biotechnology Vol 1. Amsterdam: Elsevier Science; 2001:307-345.

27. Patel T, Pandya H. Citric acid production fermentation process. Int J Adv Res Educ Technol 2017;3(2):3983-3991.

28. Sauer M, Porro D, Mattanovich D, Branduardi P. Microbial production of organic acids: expanding the markets. Trends Biotechnol 2008;26(2):100-108. doi:10.1016/j. tibtech.2007.11.006

29. Darvishi F, Nahvi I, Zarkesh-Esfahani H, Momenbeik F. Effect of plant oils upon lipase and citric acid production in 
Yarrowia lipolytica yeast. J Biomed Biotechnol 2009;2009. doi:10.1155/2009/562943

30. Kristiansen B, Mattey M, Linden J. Citric acid biotechnology. Taylor\&Francis e-Library; 2002.

31. Berovic M, Legisa M. Citric acid production. Biotechnol Annu Rev 2007;13:303-343. doi:10.1016/S13872656(07)13011-8

32. Murphy RA, Horgan KA. Antibiotics, enzymes and chemical commodities from fungi. In: Kavanagh K, ed. Fungi: Biology and Applications England: John Wiley\&Sons; 2005:113-143. doi:10.1002/0470015330.ch5

33. Demain AL, Phaff HJ, Kurtzman CP. The industrial and agricultural significance of yeasts. In: Kurtzman CP, Fell JW, eds. The yeasts, A taxonomic study 4th ed. Amsterdam: Elsevier; 1998:13-19. doi:10.1016/b978-0444813121/50006-x

34. Wojtatowicz M, Rymowicz W, Kautola H. Comparison of different strains of the yeast Yarrowia lipolytica for citric acid production from glucose hydrol. Appl Biochem Biotechnol 1991;31(2):165-174. doi:10.1007/BF02921787

35. Kamzolova S V, Lunina JN, Morgunov IG. Biochemistry of citric acid production from rapeseed oil by Yarrowia lipolytica yeast. J Am Oil Chem Soc 2011;88(12):1965-1976. doi:10.1007/s11746-011-1954-1

36. Antonucci S, Bravi M, Bubbico R, Di Michele A, Verdone N. Selectivity in citric acid production by Yarrowia lipolytica. Enzyme Microb Technol 2001;28(2-3):189-195. doi:10.1016/S0141-0229(00)00288-X

37. Holz M, Förster A, Mauersberger S, Barth G. Aconitase overexpression changes the product ratio of citric acid production by Yarrowia lipolytica. Appl Microbiol Biotechnol 2009;81(6):1087-1096. doi:10.1007/s00253-008-1725-6

38. Hesham AE-L, Mostafa YS, AlSharqi LEO. Optimization of citric acid production by immobilized cells of novel yeast isolates. Mycobiology 2020;48(2):122-132. doi:10.10 80/12298093.2020.1726854

39. Liu X-Y, Chi Z, Liu G-L, Wang F, Madzak C, Chi Z-M. Inulin hydrolysis and citric acid production from inulin using the surface-engineered Yarrowia lipolytica displaying inulinase. Metab Eng 2010;12(5):469-476. doi:10.1016/j. ymben.2010.04.004

40. Sanchez-Riera F. Production of organic acids. Biotechnology 2010;5:1-9.

41. Fickers P, Cheng H, Sze Ki Lin C. Sugar alcohols and organic acids synthesis in Yarrowia lipolytica: Where are we? Microorganisms 2020;8(4). doi:10.3390/microorganisms8040574

42. Yalçın SK, Bozdemir MT, Özbaş ZY. Fermantasyon yolu ile sitrik asit üretiminde farklı substrat kaynaklarının kullanılma olanakları. Gıda 2010;35(2):135-142.

43. Yalçın S. Investigation of various parameters affecting citric acid production by Yarrowia lipolytica and assessment of citric acid production capacities of some endogenic yeast strains. 2007; PhD Thesis, Hacettepe University.

44. Anastassiadis S, Rehm HJ. Continuous citric acid secretion by a high specific $\mathrm{pH}$ dependent active transport system in yeast Candida oleophila ATCC 20177. Electron J Biotechnol 2005;8(2):146-161. doi:10.2225/vol8-issue2-fulltext-11

45. Kamzolova S V, Morgunov IG, Aurich A, et al. Lipase secretion and citric acid production in Yarrowia lipolytica yeast grown on animal and vegetable fat. Food Technol Biotechnol 2005;43(2):113-122.

46. Rymowicz W, Rywińska A, Gładkowski W. Simultaneous production of citric acid and erythritol from crude glycerol by Yarrowia lipolytica Wratislavia K1. Chem Pap 2008;62(3):239-246. doi:10.2478/s11696-008-0018-y

47. Vandenberghe LP, Soccol C., Pandey A, Lebeault J. Microbial production of citric acid. Brazilian Arch Biol Technol 1999;42:263-276. doi:https://doi.org/10.1590/S151689131999000300001

48. Abu Yazid N, Barrena R, Komilis D, Sánchez A. Solid-state fermentation as a novel paradigm for organic waste valorization: A review. Sustain 2017;9(2):224. doi:10.3390/ su9020224

49. Imandi SB, Bandaru VVR, Somalanka SR, Bandaru SR, Garapati HR. Application of statistical experimental designs for the optimization of medium constituents for the production of citric acid from pineapple waste. Bioresour Technol 2008;99(10):4445-4450. doi:10.1016/j. biortech.2007.08.071

50. Moeller L, Strehlitz B, Aurich A, Zehnsdorf A, Bley T. Optimization of citric acid production from glucose by Yarrowia lipolytica. Eng Life Sci 2007;7(5):504-511. doi:10.1002/ elsc. 200620207

51. Morgunov IG, Kamzolova S V, Lunina JN. The citric acid production from raw glycerol by Yarrowia lipolytica yeast and its regulation. Appl Microbiol Biotechnol 2013;97(16):7387-7397. doi:10.1007/s00253-013-5054-z

52. Anastassiadis S, Rehm HJ. Oxygen and temperature effect on continuous citric acid secretion in Candida oleophila. Electron J Biotechnol 2006;9(4):414-423. doi:10.2225/ vol9-issue4-fulltext-3

53. Kubicek CP. Organic acids. In: Ratledge C, Kristiansen B, eds. Basic Biotechnology 2nd ed. Cambridge University Press; 2001:305-324.

54. Karasu-Yalcin S, Bozdemir MT, Ozbas ZY. A comparative study of the effects of glycerol and mannitol on citric acid production by two Yarrowia lipolytica strains. Rom Biotechnol Lett 2009;14(6):4870-4881.

55. Rane KD, Sims KA. Production of citric acid by Candida lipolytica Y1095: Effect of glucose concentration on yield and productivity. Enzyme Microb Technol 1993;15(8):646651. doi:10.1016/0141-0229(93)90063-8

56. Fu G-Y, Lu Y, Chi Z, et al. Cloning and characterization of a pyruvate carboxylase gene from Penicillium rubens and overexpression of the gene in the yeast Yarrowia lipolytica for enhanced citric acid production. Mar Biotechnol 2015;18(1):1-14. doi:10.1007/s10126-015-9665-5

57. Hattori K, Suzuki T. Large scale production of erythritol and its conversion tod-mannitol production by $\mathrm{n}$-al- 
kane-grown Candida zeylanoides. Agric Biol Chem 1974;38(6):1203-1208. doi:10.1080/00021369.1974.10861 309

58. Yalcin SK, Bozdemir MT, Ozbas ZY. Citric acid production by yeasts: fermentation conditions, process optimization and strain improvement. Curr Res Technol Educ Top Appl Microbiol Microb Biotechnol 2010;9:1374-1382.

59. Kim KH, Lee HY, Lee CY. Pretreatment of sugarcane molasses and citric acid production by Candida zeylanoides. Korean J Microbiol Biotechnol 2015;43(2):164-168. doi:10.4014/mbl.1503.03006

60. Hamissa FA, Abou-Zeid A-ZA, Redwan AA. Fermentative production of citric acid by yeasts. Agric Wastes 1981;3(1):21-33. doi:10.1016/0141-4607(81)90004-4

61. Demain AL, Sanchez S. Microbial synthesis of primary metabolites: Current trends and future prospects. In: El-Mansi EM., Bryce CF., Dahhou B, Sanchez S, Demain A., Allman A., eds. Fermentation Microbiology and Biotechnology 3th ed. Boca Raton, FL: CRC Press; 2012:77100.

62. Souza KST, Schwan RF, Dias DR. Lipid and citric acid production by wild yeasts grown in glycerol. J Microbiol Biotechnol 2014;24(4):497-506. doi:10.4014/jmb.1310.10084

63. Kieliszek M, Kot AM, Bzducha-Wróbel A, BŁażejak S, Gientka I, Kurcz A. Biotechnological use of Candida yeasts in the food industry: A review. Fungal Biol Rev 2017;31(4):185-198. doi:10.1016/j.fbr.2017.06.001

64. Carsanba E, Papanikolaou S, Fickers P, Erten H. Screening various Yarrowia lipolytica strains for citric acid production. Yeast 2019;36(5):319-327. doi:10.1002/yea.3389

65. Papanikolaou S, Galiotou-Panayotou M, Chevalot I, Komaitis M, Marc I, Aggelis G. Influence of glucose and saturated free-fatty acid mixtures on citric acid and lipid production by Yarrowia lipolytica. Curr Microbiol 2006;52(2):134-142. doi:10.1007/s00284-005-0223-7

66. Liu X, Lv J, Xu J, Zhang T, Deng Y, He J. Citric acid production in Yarrowia lipolytica SWJ-1b yeast when grown on waste cooking oil. Appl Biochem Biotechnol 2015;175(5):2347-2356. doi:10.1007/s12010-014-1430-0

67. Förster A, Aurich A, Mauersberger S, Barth G. Citric acid production from sucrose using a recombinant strain of the yeast Yarrowia lipolytica. Appl Microbiol Biotechnol 2007;75(6):1409-1417. doi:10.1007/s00253-007-0958-0

68. Kamzolova SV, Shamin RV, Stepanova NN, Morgunov GI, Lunina JN, Allayarov RK, Samoilenko VA, Morgunov IG. Fermentation conditions and media optimization for isocitric acid production from ethanol by Yarrowia lipolytica. Biomed Res Int 2018;2018. doi:10.1155/2018/2543210

69. Ferreira P, Lopes M, Mota M, Belo I. Oxygen mass transfer impact on citric acid production by Yarrowia lipolytica from crude glycerol. Biochem Eng J 2016;110:35-42. doi:10.1016/j.bej.2016.02.001

70. Rehm HJ, Reed G, Pühler A, Stadler P. Citric acid. In: Roehr M, Kubicek C., Kominek J, eds. Biotechnology Vol 6. 2nd ed. Germany: VCH Publishers; 1996:307-346.
71. Crolla A, Kennedy KJ. Fed-batch production of citric acid by Candida lipolytica grown on n-paraffins. J Biotechnol 2004;110(1):73-84. doi:10.1016/j.jbiotec.2004.01.007

72. Rywińska A, Musiał I, Rymowicz W, Żarowska B, Boruczkowski T. Effect of agitation and aeration on the citric acid production by Yarrowia lipolytica grown on glycerol. Prep Biochem Biotechnol 2012;42(3):279-291. do i: $10.1080 / 10826068.2012 .656868$

73. Sabra W, Bommareddy RR, Maheshwari G, Papanikolaou S, Zeng AP. Substrates and oxygen dependent citric acid production by Yarrowia lipolytica: insights through transcriptome and fluxome analyses. Microb Cell Fact 2017;16(1):78. doi:10.1186/s12934-017-0690-0

74. Finogenova TV, Kamzolova SV, Dedyukhina EG, Shishkanova NV, Il'Chenko AP, Morgunov IG, Chernyavskaya OG, Sokolov AP. Biosynthesis of citric and isocitric acids from ethanol by mutant Yarrowia lipolytica N 1 under continuous cultivation. Appl Microbiol Biotechnol 2002;59(4):493-500. doi:10.1007/s00253-002-1022-8

75. Pazouki M, Felse PA, Sinha J, Panda T. Comparative studies on citric acid production by Aspergillus niger and Candida lipolytica using molasses and glucose. Bioprocess Eng 2000;22(4):353-361. doi:10.1007/PL00009115

76. Arzumanov TE, Shishkanova N V., Finogenova T V. Biosynthesis of citric acid by Yarrowia lipolytica repeat-batch culture on ethanol. Appl Microbiol Biotechnol 2000;53(5):525-529. doi:10.1007/s002530051651

77. Karasu-Yalcin S, Tijen Bozdemir M, Yesim Ozbas Z. Effects of different fermentation conditions on growth and citric acid production kinetics of two Yarrowia lipolytica strains. Chem Biochem Eng Q 2010;24(3):347-360.

78. Good DW, Droniuk R, Lawford GR, Fein JE. Isolation and characterization of a Saccharomycopsis lipolytica mutant showing increased production of citric acid from canola oil. Can J Microbiol 1985;31(5):436-440. doi:10.1139/ m85-081

79. Anastassiadis S, Wandrey C, Rehm HJ. Continuous citric acid fermentation by Candida oleophila under nitrogen limitation at constant $\mathrm{C} / \mathrm{N}$ ratio. World J Microbiol Biotechnol 2005;21(5):695-705. doi:10.1007/s11274-0043850-4

80. Briffaud J, Engasser J. Citric acid production from glucose. II. Growth and excretion kinetics in a trickleflow fermentor. Biotechnol Bioeng 1979;21(11):2083-2092. doi:10.1002/bit.260211114

81. Kamzolova S V., Morgunov IG. Metabolic peculiarities of the citric acid overproduction from glucose in yeasts Yarrowia lipolytica. Bioresour Technol 2017;243:433-440. doi:10.1016/j.biortech.2017.06.146

82. Mafakher L, Mirbagheri M, Darvishi F, Nahvi I, Zarkesh-Esfahani H, Emtiazi G. Isolation of lipase and citric acid producing yeasts from agro-industrial wastewater. N Biotechnol 2010;27(4):337-340. doi:10.1016/j. nbt.2010.04.006

83. Moeller L, Grünberg M, Zehnsdorf A, Strehlitz B, Bley 
T. Biosensor online control of citric acid production from glucose by Yarrowia lipolytica using semicontinuous fermentation. Eng Life Sci 2010;10(4):311-320. doi:10.1002/elsc.200900073

84. Rymowicz W, Rywińska A, Żarowska B, Juszczyk P. Citric acid production from raw glycerol by acetate mutants of Yarrowia lipolytica. Chem Pap 2006;60(5):391394. doi:10.2478/s11696-006-0071-3

85. Rywińska A, Juszczyk P, Wojtatowicz M, Rymowicz W. Chemostat study of citric acid production from glycerol by Yarrowia lipolytica. J Biotechnol 2011;152(1-2):5457. doi:10.1016/j.jbiotec.2011.01.007

86. Tomaszewska L, Rakicka M, Rymowicz W, Rywińska A.A comparative study on glycerol metabolism to erythritol and citric acid in Yarrowia lipolytica yeast cells. FEMS Yeast Res 2014;14(6):966-976. doi:10.1111/15671364.12184

87. Lazar Z, Walczak E, Robak M. Simultaneous production of citric acid and invertase by Yarrowia lipolytica SUC+ transformants. Bioresour Technol 2011;102(13):69826989. doi:10.1016/j.biortech.2011.04.032

88. Papanikolaou S, Muniglia L, Chevalot I, Aggelis G, Marc I. Yarrowia lipolytica as a potential producer of citric acid from raw glycerol. J Appl Microbiol 2002;92(4):737-744. doi:10.1046/j.1365-2672.2002.01577.x

89. Papanikolaou S, Fakas S, Fick M, et al. Biotechnological valorisation of raw glycerol discharged after bio-diesel (fatty acid methyl esters) manufacturing process: Production of 1,3-propanediol, citric acid and single cell oil. Biomass and Bioenergy 2008;32(1):60-71. doi:10.1016/j. biombioe.2007.06.007

90. Levinson WE, Kurtzman CP, Kuo TM. Characterization of Yarrowia lipolytica and related species for citric acid production from glycerol. Enzyme Microb Technol 2007;41(3):292-295. doi:10.1016/j. enzmictec.2007.02.005

91. Makri A, Fakas S, Aggelis G. Metabolic activities of biotechnological interest in Yarrowia lipolytica grown on glycerol in repeated batch cultures. Bioresour Technol 2010;101(7):2351-2358. doi:10.1016/j. biortech.2009.11.024

92. André A, Chatzifragkou A, Diamantopoulou P, et al. Biotechnological conversions of bio-dieselderived crude glycerol by Yarrowia lipolytica strains. Eng Life Sci 2009;9(6):468-478. doi:10.1002/elsc.200900063

93. Imandi SB, Bandaru VR, Somalanka SR, Garapati HR. Optimization of medium constituents for the production of citric acid from byproduct glycerol using Doehlert experimental design. Enzyme Microb Technol 2007;40(5):1367-1372. doi:10.1016/j. enzmictec.2006.10.012

94. Rymowicz W, Fatykhova AR, Kamzolova S V, Rywińska A, Morgunov IG. Citric acid production from glycerol-containing waste of biodiesel industry by Yarrowia lipolytica in batch, repeated batch, and cell recycle re- gimes. Appl Microbiol Biotechnol 2010;87(3):971-979. doi:10.1007/s00253-010-2561-z

95. Kamzolova SV., Fatykhova AR, Dedyukhina EG, Anastassiadis SG, Golovchenko NP, Morgunov IG. Citric acid production by yeast grown on glycerol-containing waste from biodiesel industry. Food Technol Biotechnol 2011;49(1):65-74.

96. Kamzolova S V, Finogenova T V, Morgunov IG. Microbiological production of citric and isocitric acids from sunflower oil. Food Technol Biotechnol 2008;46(1):5159.

97. Venter T, Kock JLF, Botes PJ, Smit MS, Hugo A, Joseph M. Acetate Enhances citric acid production by Yarrowia lipolytica when grown on sunflower oil. Syst Appl Microbiol 2004;27(2):135-138. doi:10.1078/072320204322881736

98. Çelik G, Bahriye Uçar F, Akpınar O, Çorbacı C. Production of citric and isocitric acid by Yarrowia lipolytica strains grown on different carbon sources. Turkish J Biochem 2014;39(3):285-290. doi:10.5505/tjb.2014.92005

99. Arslan NP, Aydogan MN, Taskin M. Citric acid production from partly deproteinized whey under non-sterile culture conditions using immobilized cells of lactose-positive and cold-adapted Yarrowia lipolytica B9. J Biotechnol 2016;231:32-39. doi:10.1016/j.jbiotec.2016.05.033

100. Yalcin SK, Bozdemir MT, Ozbas ZY. Utilization of whey and grape must for citric acid production by two Yarrowia lipolytica strains. Food Biotechnol 2009;23(3):266283. doi:10.1080/08905430903106860

101. Yalcin SK, Bozdemir MT, Ozbas ZY. A comparative study on citric acid production kinetics of two Yarrowia lipolytica strains in two different media. Indian J Biotechnol 2009;8:408-417.

102. Papanikolaou S, Galiotou-Panayotou M, Fakas S, Komaitis M, Aggelis G. Citric acid production by Yarrowia lipolytica cultivated on olive-mill wastewater-based media. Bioresour Technol 2008;99(7):2419-2428. doi:10.1016/j.biortech.2007.05.005

103. Sarris D, Galiotou-Panayotou M, Koutinas AA, Komaitis M, Papanikolaou S. Citric acid, biomass and cellular lipid production by Yarrowia lipolytica strains cultivated on olive mill wastewater-based media. J Chem Technol Biotechnol 2011;86(11):1439-1448. doi:10.1002/ jctb. 2658

104. Sarris D, Stoforos NG, Mallouchos A, Kookos IK, Koutinas AA, Aggelis G, Papanikolaou S. Production of added-value metabolites by Yarrowia lipolytica growing in olive mill wastewater-based media under aseptic and non-aseptic conditions. Eng Life Sci 2017;17(6):695709. doi:10.1002/elsc.201600225 